\title{
Combination dolutegravir-abacavir-lamivudine in the management of HIV/AIDS: clinical utility and patient considerations
}

This article was published in the following Dove Press journal:

Patient Preference and Adherence

17 February 2015

Number of times this article has been viewed

\author{
Mario Cruciani \\ Marina Malena \\ Center of Community Medicine \\ and HIV Outpatient Clinic, Infectious \\ Diseases Unit, San Bonifacio Hospital, \\ Verona, Italy
}

\begin{abstract}
The current standard of care for human immunodeficiency virus (HIV) treatment is a three-drug regimen containing a nonnucleoside reverse transcriptase inhibitor, a protease inhibitor, or an integrase strand transfer inhibitor (INSTI) plus two nucleoside/tide reverse transcriptase inhibitors. Given their potency, safety, and distinctive mechanism of action, INSTIs represent an important advance in HIV type 1 (HIV-1) therapy. Dolutegravir (DTG) is a new-generation INSTI recently approved for the treatment of HIV-1-infected adult patients, with distinct advantages compared with other available antiretroviral agents. In well-designed, large clinical trials, DTG-containing regimens have demonstrated either noninferiority or superiority to current first-line agents such as raltegravir-, darunavir/ritonavir-, and efavirenz-containing regimens. The favorable safety profile, low potential for drug interactions, minimal impact on lipids, good tolerability, and high resistance barrier of DTG makes this compound one of the preferred choices for HIV therapy in multiple clinical scenarios, including treatment-naïve and treatment-experienced patients. DTG is the only antiretroviral drug not yet associated with de novo emergence of resistance mutations in treatment-naïve individuals. However, data from in vitro studies and clinical trial suggest the possibility of cross-resistance between first- and second-generation INSTIs. Even though these profiles are infrequent at the moment, they need to be monitored in all current patients treated with INSTIs. With its potent activity, good tolerability, simplicity of dosing, and minimal drug interaction profile, DTG will likely play a major role in the management of patients with HIV-1 infection. On the basis of clinical trial data, current guidelines endorse DTG in combination with nucleoside/tide reverse transcriptase inhibitors as one of the recommended regimens in antiretroviral therapy-naïve patients. Most of the favorable clinical experiences from clinical trials are based on the combination of DTG with abacavir/lamivudine, and DTG is planned to be coformulated with abacavir/lamivudine. This will provide a further advantage, given that single tablet regimens are associated with higher adherence rates as well as improvement in quality of life and enhanced patient preference.
\end{abstract}

Keywords: HIV-1, antiretroviral agents, dolutegravir, abacavir/lamivudine, review

\section{Introduction}

The treatment of human immunodeficiency virus (HIV) infection has profoundly changed after the availability of combined antiretroviral therapy (cART). Use of these multidrug regimens has resulted in substantial reductions in progression to acquired immune deficiency syndrome (AIDS), opportunistic infections, hospitalizations, and death. However, drug selection is becoming increasingly complex, with more than 25 antiretroviral medications available in six major classes, including nucleoside/ nucleotide reverse transcriptase inhibitors (NRTIs), nonnucleoside reverse transcriptase
Correspondence: Mario Cruciani

Center of Community Medicine and HIV Outpatient Clinic, ULSS 20 Verona, Via Germania, 20-37I35 Verona, Italy Email crucianimario@virgilio.it submit your manuscript | www.dovepress.com

Dovepress

http://dx.doi.org// 0.2147/PPA.S65199 
inhibitors (NNRTIs), protease inhibitors (PIs), fusion inhibitors, CCR5 antagonists, and integrase strand transfer inhibitors (INSTIs). ${ }^{1,2}$

The current standard of care for HIV treatment is a threedrug regimen containing an NNRTI, a PI, or an INSTI plus two NRTIs. ${ }^{1,2}$

Factors influencing the choice between an NNRTI, a PI, or an INSTI as the third drug in a regimen include efficacy, safety, tolerability, convenience for the patient (eg, dosing frequency, number of pills, food requirements), genetic barriers to resistance, comorbidities, and potential for drug-drug interactions.

The aim of this review is to evaluate the clinical utility of the combination of dolutegravir-abacavir-lamivudine (DTG/ABC/3TC) in the management of HIV type 1 (HIV-1) infection. Since it is likely that DTG use in the near future will be mostly represented in coformulation with an NRTI backbone, we will also focus on comparative efficacy and safety aspects of NRTIs.

\section{Methods}

The literature used in this traditional review extended up to December 2014. For NRTIs, we updated the search criteria already used in recent systematic reviews on the safety and efficacy of ABC compared with other NRTIs conducted by our group. ${ }^{3}$ For DTG and other INSTIs, we selected and summarized the most relevant papers related to the safety and efficacy of INSTIs. For this purpose, we searched PubMed using various combinations of the following terms: HIV, integrase inhibitors, raltegravir (RAL), DTG, elvitegravir (EVG), clinical trials, and randomized clinical trials (RCTs).

\section{Choosing among NRTIs}

NRTIs have historically been the cornerstones of HIV therapy since the first NRTI was introduced in 1987. Currently recommended regimens include the NRTI combination of tenofovir disoproxil fumarate/emtricitabine (TDF/FTC) or $\mathrm{ABC} / 3 \mathrm{TC}$, both available as fixed-dose combination tablets given once daily. Factors to be considered when choosing between these NRTI combinations are related to safety and virologic efficacy.

Current guidelines assume 3TC and FTC to be equivalent. ${ }^{1}$ Recently, an analysis from a treatment cohort in the Netherlands suggests better virologic response to FTC compared with lamivudine as part first-line ART. ${ }^{4}$ However, this cohort study was greatly unbalanced. Patients in the lamivudine group had a median ART initiation year at 2004 (compared with 2009 for FTC), had a higher baseline viral load
(VL) and lower baseline CD4 cell counts, were more likely to be injecting drug users (which could influence adherence), and were more likely to be coinfected with hepatitis B. Thus, in light of these methodological limitations and the large discrepancy between the results of this cohort study and those provided by prospective RCTs, it is reasonable to believe that the observed treatment differences are the result of study design flaws rather than true differences in efficacy between lamivudine and FTC. ${ }^{5}$

Compared with lamivudine, FTC shows a longer plasma and intracellular triphosphate half-life. The results of a recent meta-analysis of clinical trials comparing FTC with lamivudine as part of a combination regimen have demonstrated that the two compounds are clinically equivalent. ${ }^{6}$ Thus, in clinical practice, the choice of FTC versus lamivudine will most likely be made in the context of their coformulation partner.

Differences between ABC and TDF are of more interest. While TDF has few initial treatment-limiting initial side effects, older studies with $\mathrm{ABC}$ are difficult to interpret due to the excess of $\mathrm{ABC}$ hypersensitivity reactions, which lead to early discontinuation and hence an excess of "failure" in intention-to-treat analysis. The establishment of HLA-B*5701 testing has largely led to the abolition of this adverse effect from clinical practice. ${ }^{7-9}$

An association between $\mathrm{ABC}$ exposure and risk of myocardial infarction has been observed in the D:A:D study, ${ }^{10-13}$ an observational cohort of studies of adverse events among patients receiving ART. Potential confoundings not adjusted for in the D:A:D study have been proposed, including prescription bias to $\mathrm{ABC}$ in patients with metabolic syndromes, lipoatrophy, dyslipidemia, renal disease, and chronic heart disease. ${ }^{14}$ A significant independent association between decreased kidney function and increased risk of cardiovascular events in HIV-1-infected patients has been reported. ${ }^{15}$

Observational studies are useful tools in pharmacovigilance when significant barriers to the conduct of RCTs exist, such as the requirement for a very large sample size or a prolonged follow-up. However, there is controversy over the validity of nonrandomized evidence, related to the existence and magnitude of selection bias. ${ }^{16}$ Actually, no excess risk of myocardial infarction with $\mathrm{ABC}$ therapy has been observed in the aggregated clinical trials database maintained by the manufacturer of $\mathrm{ABC}$ and in two independent meta-analyses of RCTs comparing ABC-containing cART with other regimens not containing $\mathrm{ABC} .{ }^{17-19}$ The majority of studies, both observational and randomized, conducted in the absence of confounding factors have not demonstrated an 
increased ABC-attributable cardiovascular risk. ${ }^{20-22}$ To date, many hypotheses and in vitro data investigating biological mechanisms to explain a potential increase in the risk of cardiovascular diseases in $\mathrm{ABC}$ recipients have been produced, but the topic remains controversial, and none of the hypotheses has been established or clinically proven. ${ }^{23}$

While ABC has been linked to cardiovascular diseases, a number of reports have linked TDF to renal tubular dysfunction, acute renal failure, and decreases in bone density and hypophosphatemia. ${ }^{1}$ In a comparative safety study of $\mathrm{ABC} / 3 \mathrm{TC}$ and TDF/FTC, differences in tubular protein loss, bone turnover markers, and declines in bone density were observed favoring ABC/3TC. ${ }^{24}$ TDF can cause nephrotoxicity, particularly in patients who have pre-existing renal disease or risk factors for kidney disease (eg, use of other nephrotoxic drugs) or in those receiving PIs or colbicistat. ${ }^{1}$

Beyond safety issues, the comparative virologic efficacy of the two NRTI backbone combinations has been the subject of recent debate. An RCT (ACTG 5202) showed that among patients with a high VL at study entry (screening HIV ribonucleic acid [RNA] $>100,000$ copies $/ \mathrm{mL}$ ), those taking $\mathrm{ABC} / 3 \mathrm{TC}$ with either boosted atazanavir (ATV) or efavirenz (EFV) were more likely to experience virologic failure than those taking TDF/FTC. ${ }^{25}$ Of note, ACTG 5202 has specific end points (week 16 definitions of viral failure, plus later ones) not used in other studies, and lack HLAB*5701 testing at baseline; moreover, the "as treated" analysis (where switch for any reason was not treated as failure) did not show the "discordant" effect, and rates of suppression were similar regardless of baseline VL. This suggests an anomaly arising from the early end point definitions in ACTG 5202 over classifying $\mathrm{ABC}$ recipients as failures. By contrast, an RCT with a head-to-head comparison of ABC/3TC with TDF/FTC, in combination with lopinavir/ritonavir, and a retrospective analysis of six previous $\mathrm{ABC}$ trials found that the backbones had similar efficacy. ${ }^{26,27}$

Numerous RCTs have been conducted to assess the comparative virologic efficacy of $\mathrm{ABC} / 3 \mathrm{TC}$ and $\mathrm{TDF} / \mathrm{FTC}$, and the results of these studies have been the object of a recent systematic review and meta-analysis. ${ }^{3}$ Meta-analytical pooling of RCTs with a direct comparison of ABC/3TC and TDF/FTC according to baseline VL at 48 weeks and 96 weeks showed that rates of patients with $\mathrm{VL}<50$ copies/mL were similar in the overall comparison, in the low baseline VL strata $(<100,000$ copies/mL), and in the high baseline VL strata $(>100,000$ copies/mL). Differences in the occurrence of adverse events requiring discontinuation of treatment favored TDF recipients, but this difference, mostly related to suspected $\mathrm{ABC}$ hypersensitivity reaction, was not statistically significant. In conclusion, the findings of this meta-analysis suggest a similar virologic efficacy of $\mathrm{ABC} / 3 \mathrm{TC}$ and TDF/ FTC regardless of the baseline VL.

No differences in time to virologic failure were observed in a large Canadian cohort of HIV-infected individuals. ${ }^{28}$ The time to regimen failure was similar for $\mathrm{ABC} / 3 \mathrm{TC}$ and TDF/FTC even when stratified by baseline VL $(<100,000$ or $>100,000$ copies $/ \mathrm{mL}$ ).

In the SINGLE trial, ${ }^{29}$ in which DTG plus ABC/3TC was compared with EFV/TDF/FTC, there was no difference in virologic responses between $\mathrm{TDF} / \mathrm{FTC}$ and $\mathrm{ABC} / 3 \mathrm{TC}$ in patients with high HIV RNA levels.

Altogether, these results support the use of either NRTI backbone in the initial therapy of ART-naïve patients and more generally of $\mathrm{ABC} / 3 \mathrm{TC}$ as one of the preferred NRTI options. . $^{2,3,28}$

\section{Choosing among INSTI options}

Given their potency, safety, and distinctive mechanism of action, INSTIs represent an important advance in HIV-1 therapy. After entry into $\mathrm{CD}^{+}{ }^{+} \mathrm{T}$-cells, viral RNA is transcribed into DNA by HIV reverse transcriptase; following this step, DNA of HIV is incorporated into the DNA of CD4 cells, a process known as integration. Integration occurs in three steps: formation of a preintegration viral DNA complex, $3^{\prime}$ processing, and strand transfer. ${ }^{30-33}$ To date, all INSTIs approved for clinical use specifically target the second step, strand transfer, of the integration process. These drugs prevent or inhibit the binding of the preintegration complex to host cell DNA, thus terminating the integration step of HIV replication. INSTIs available for clinical use include RAL, EVG, and DTG.

RAL is the first INSTI approved for clinical use. ${ }^{1,2,30,34}$ Clinical trials have demonstrated that RAL-containing regimens have potent antiretroviral activity and are well tolerated..$^{35-38}$ In addition, because of their unique mode of action, INSTIs retain activity against isolates that have acquired resistance to other classes of antiretroviral agents.

The BENCHMRK studies demonstrated that the use of RAL was highly effective in achieving viral suppression when combined with an optimized background regimen in treatment-experienced patients with drug-resistant HIV infection. ${ }^{35,36}$

In treatment-naïve persons, the efficacy of RAL was evaluated in the STARTMRK trials, which randomly assigned previously untreated $\mathrm{HIV}$-infected individuals to receive twice-daily RAL or once-daily EFV, each in 
combination with fixed-dose TDF/FTC. ${ }^{37,38}$ RAL produced durable viral suppression and immune restoration that were at least equivalent to those of EFV through 156 weeks of therapy. Both regimens were well tolerated, but RAL was associated with fewer drug-related clinical adverse events and smaller elevations in lipid levels. As was noted in early studies, ${ }^{30,34}$ treatment with RAL was associated with faster virologic response.

The advantages of RAL include potent antiviral activity, limited drug-drug interactions, excellent tolerability, and a long postmarketing experience..$^{1,30,35-40}$ However, limitations include its twice-daily dosing schedule and a low genetic barrier to viral resistance. . $^{1,30}$

EVG was recently approved for clinical use as a oncea-day combination tablet coformulated with cobicistat (COBI) and TDF/FTC (EVG/COBI/TDF/FTC). EVG/ $\mathrm{COBI} / \mathrm{TDF} / \mathrm{FTC}$ was noninferior to $\mathrm{EFV} / \mathrm{TDF} / \mathrm{FTC}$ and to ritonavir-boosted ATV in combination with TDF/FTC in treatment-naïve patients at 48 and 96 weeks. ${ }^{41-44}$ One of the disadvantages of EVG is its short half-life, which necessitates the administration of the pharmacokinetic booster COBI. COBI is a specific and potent inhibitor of CYP3A4 and is associated with clinically significant drug-drug interactions. ${ }^{45}$ Moreover, coformulated EVG/COBI/TDF/FTC is not recommended for patients with creatinine clearance $<70 \mathrm{~mL} / \mathrm{min}$ and should be discontinued if creatinine clearance declines below $50 \mathrm{~mL} / \mathrm{min}^{1,46}$

\section{DTG, a second-generation INSTI}

DTG is the most recently approved INSTI. It is approved for use in ART-naïve and ART-experienced patients. Several clinical trials conducted in ART-naïve and ART-experienced patients have assessed the efficacy and safety of DTG, in combination with $\mathrm{ABC} / 3 \mathrm{TC}$, as well as with other NRTI backbones (Tables 1 and 2). ${ }^{47-56}$

\section{Drug efficacy}

The efficacy of DTG in treatment-naïve patients has been evaluated in three fully powered clinical trials, including two randomized double-blind clinical trials and one randomized open-label clinical trial. ${ }^{48-53}$ In these three trials, DTG-based regimens demonstrated either noninferiority or superiority to a comparator INSTI-, NNRTI-, or PIbased regimen. The primary efficacy end point in all these clinical trials was the proportion of participants with plasma $\mathrm{VL}<50$ copies $/ \mathrm{mL}$.

The SPRING-1 study was a randomized, partially blinded, Phase IIb dose-ranging trial that assessed the 48- and 96-week

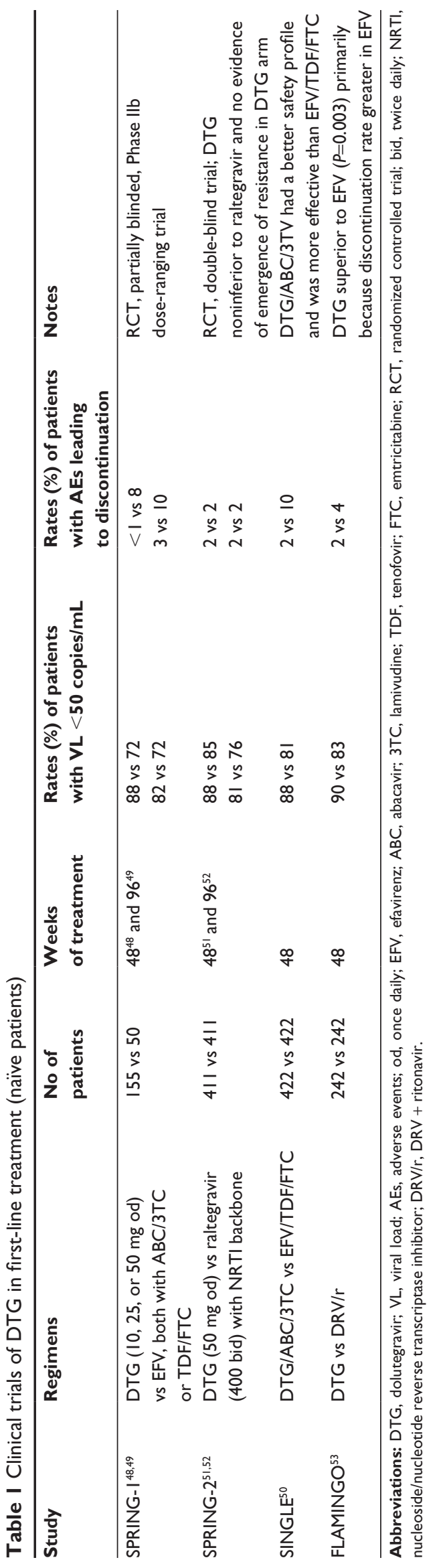


Table 2 Clinical trials of DTG in second-line treatment (ARV-experienced patients, INSTI naïve or experienced)

\begin{tabular}{|c|c|c|c|c|c|c|}
\hline Study & Regimens & $\begin{array}{l}\text { No of } \\
\text { patients }\end{array}$ & $\begin{array}{l}\text { Weeks of } \\
\text { treatment }\end{array}$ & $\begin{array}{l}\text { Rates }(\%) \text { of patients } \\
\text { with } \mathbf{V L}<\mathbf{5 0} \\
\text { copies } / \mathbf{m L}\end{array}$ & $\begin{array}{l}\text { Rates (\%) of patients } \\
\text { with AEs leading } \\
\text { to discontinuation }\end{array}$ & Notes \\
\hline SAILING ${ }^{54}$ & $\begin{array}{l}\text { DTG } 50 \mathrm{mg} \\
\text { vs raltegravir } \\
400 \text { bid + OBT }\end{array}$ & 354 vs $36 I$ & 48 & $7 I$ vs 64 & I vs I & $\begin{array}{l}\text { INSTI-naïve patients; } \\
\text { RCT, double blind }\end{array}$ \\
\hline $\begin{array}{l}\text { VIKING } \\
(\text { Cohort I) })^{55}\end{array}$ & $\begin{array}{l}\text { DTG } 50 \mathrm{mg} \\
\text { od + OBT }\end{array}$ & 27 & 24 & $4 I$ & $\begin{array}{l}\text { No severe AE related } \\
\text { to } D T G \text { reported }\end{array}$ & $\begin{array}{l}\text { Phase Ilb, open label, } \\
\text { single arm; INSTI } \\
\text { resistant }\end{array}$ \\
\hline $\begin{array}{l}\text { VIKING } \\
(\text { Cohort II) })^{55}\end{array}$ & $\begin{array}{l}\text { DTG } 50 \mathrm{mg} \\
\text { bid + OBT }\end{array}$ & 24 & 24 & 75 & $\begin{array}{l}\text { No severe AE related } \\
\text { to } D T G \text { reported }\end{array}$ & $\begin{array}{l}\text { Phase Ilb, open label, } \\
\text { single arm; INSTI } \\
\text { resistant }\end{array}$ \\
\hline VIKING-356 & $\begin{array}{l}\text { DTG } 50 \mathrm{mg} \\
\mathrm{bid}+\text { OBT }\end{array}$ & 183 & 24 & 69 & 2.7 & $\begin{array}{l}\text { Phase III, open label, } \\
\text { single arm; INSTI } \\
\text { resistant }\end{array}$ \\
\hline
\end{tabular}

Abbreviations: DTG, dolutegravir; VL, viral load; AEs, adverse events; bid, twice daily; OBT, optimized background therapy; INSTI, integrase strand transfer inhibitor; RCT, randomized controlled trial; od, once daily.

efficacy and safety of different doses of DTG $(10,25$, or $50 \mathrm{mg}$ once daily) in combination with two open-label NRTIs (selected by investigators) using EFV as comparator. ${ }^{48,49}$ No participants on DTG who met the definition for virologic failure had emergence of an INSTI mutation. ${ }^{49}$

The SINGLE trial was a Phase III trial that assessed the efficacy and safety of a regimen containing $\mathrm{ABC} / 3 \mathrm{TC}$ and DTG in comparison with EFV/TDF/FTC in 833 ART-naïve patients. ${ }^{50}$ The comparative efficacy data of the two regimens according to strata and subgroups are summarized in Figure 1. At 48 weeks, $88 \%$ of patients receiving DTG and $81 \%$ on EFV had plasma HIV RNA $<50$ copies/mL. Although initially designed as a noninferiority trial, the statistical analysis concluded that the DTG arm was superior to EFV/TDF/FTC ( $P=0.003)$, primarily because the study treatment discontinuation rate was higher in the EFV arm than in the DTG arm. Patients in the DTG arm also experienced significantly shorter median times to viral suppression (28 vs 84 days, $P<0.0001)$, as well as greater increases in CD4 counts (267 vs 208 cells $/ \mu \mathrm{L}$, respectively; $P<0.001$ ).

SPRING-2 was a randomized, double-blind, noninferiority study that compared the efficacy and safety of DTG $50 \mathrm{mg}$ once daily versus RAL $400 \mathrm{mg}$ twice daily in ART-naïve patients. ${ }^{51,52}$ The NRTI backbone (ABC/3TC or TDF/FTC) was chosen by the study investigators. At 48 weeks, $88 \%$ of patients on DTG had undetectable plasma HIV RNA in comparison with $85 \%$ in the RAL arm, thus fitting the noninferiority goal. On the other hand, no significant differences were found comparing 3TC/ABC and TDF/FTC, although the latter tended to exhibit some virologic advantage over time. At week 96, once-daily DTG was noninferior to twice-daily RAL. The authors concluded that once-daily
DTG is an attractive treatment option for HIV-1-infected treatment-naïve patients.

The efficacy of DTG versus ritonavir-boosted darunavir (DRV) (DRV/r) was compared in the FLAMINGO trial..$^{53}$ This was a multicenter, open-label, noninferiority trial that recruited ART-naïve patients and compared DTG $50 \mathrm{mg}$ once daily to DRV/r 800/100 mg once daily, each in combination with investigator-selected $\mathrm{ABC} / 3 \mathrm{TC}$ or TDF/FTC. At 48 weeks, a significantly higher proportion of patients on DTG than DRV reached undetectable plasma HIV RNA (90\% vs $83 \%, P=0.025)$. No significant differences were found among patients in the DTG arm receiving different NRTI backbones.

It should be noted that the SINGLE and FLAMINGO trials are the only trials conducted so far that have demonstrated an alternative ART regimen be superior, respectively, to EFV- and DRV-based combinations within 1-2 years (RAL was found to be superior to EFV only after 5 years in STARTMRK). ${ }^{57}$ The recognition of this greater antiviral potency of the newest drugs with respect to the classically unbeaten EFV has been highlighted by some experts as the beginning of a new ART era because of the advent of safer and more potent antiretroviral third agents. ${ }^{58}$

The efficacy ofDTG was also assessed in ART-experienced patients. DTG demonstrated efficacy in three trials conducted in ART-experienced participants, including those with resistance to RAL and EVG. ${ }^{54-56}$ The SAILING trial was a Phase III, randomized, double-blind, noninferiority study that examined the efficacy and safety of DTG versus RAL in ART-experienced, INSTI-naïve patients with resistance to two or more classes of ART and one to two fully active agents available for background therapy. ${ }^{54} \mathrm{~A}$ significantly 
Participants with HIV-1 RNA level $<50$ copies $/ \mathrm{mL}$

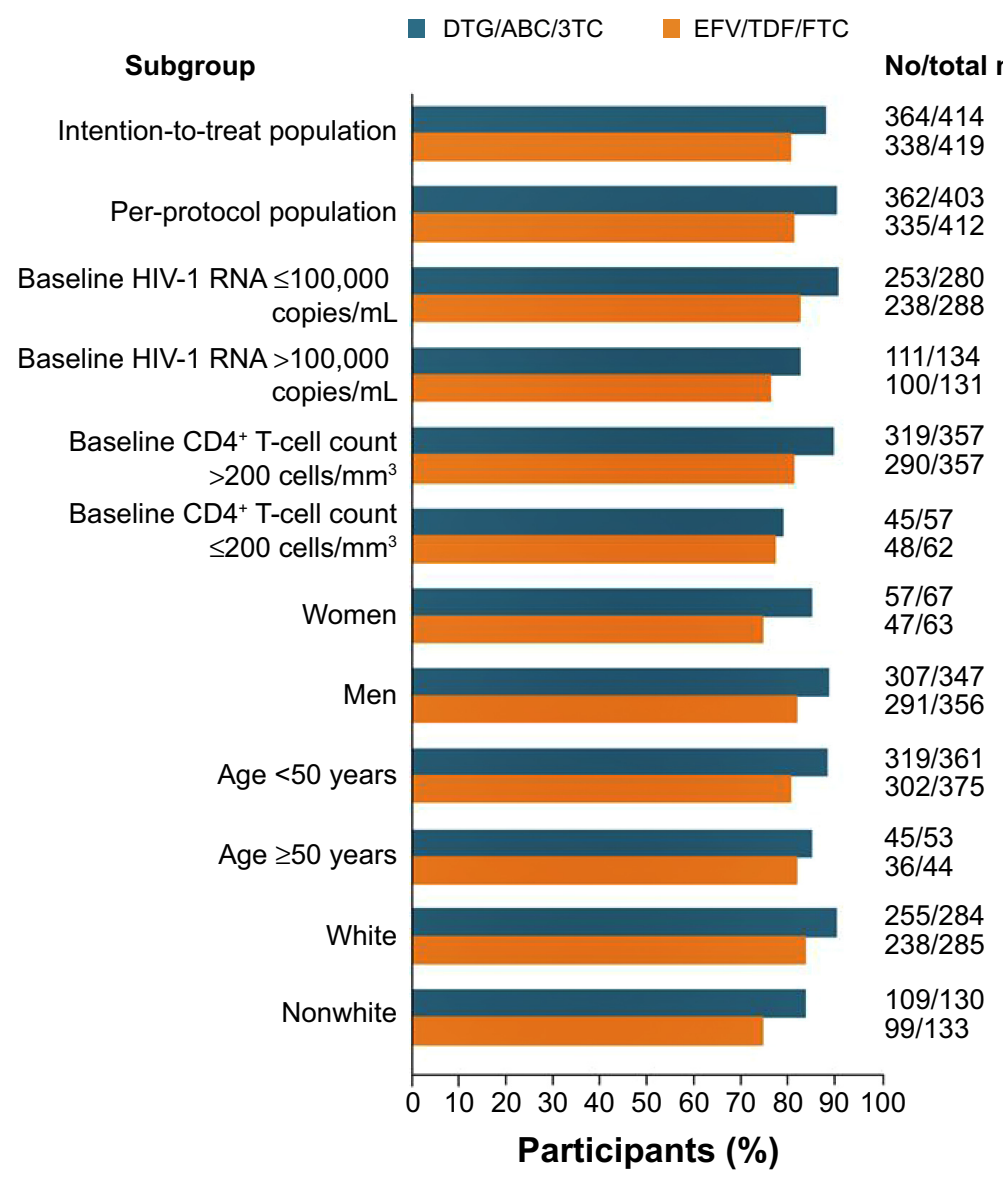

Percent difference between groups (95\% Cl)

Figure I Comparative efficacy data of EFV/TDF/FTC and DTG/ABC/3TC at week 48, according to strata and subgroups. Data from. ${ }^{50}$

Abbreviations: EFV, efavirenz; TDF, tenofovir; FTC, emtricitabine; DTG, dolutegravir; ABC, abacavir; 3TC, lamivudine; Cl, confidence interval; HIV-I, human immunodeficiency virus type I; RNA, ribonucleic acid.

higher proportion of patients on DTG than RAL achieved undetectable plasma HIV RNA at 48 weeks. The difference in response was driven by virologic outcomes, as discontinuations and adverse event rates were similar between the two groups. When stratified by VL $>50,000$ copies $/ \mathrm{mL}$, rates of patients achieving viral suppression were higher in the DTG group compared with the RAL group ( $62 \%$ vs $47 \%$, difference $15.2 \%$ [95\% confidence interval 1.9-28.4]). Fewer participants in the DTG arm with viral failure had treatment-emergent genotypic or phenotypic INSTI resistance by week 48 ( $1 \%$ in DTG vs $5 \%$ in RAL group, $P=0.003$ ). RAL-associated significant genotypic resistance was demonstrated in $42 \%$ of the participants with viral failure in the RAL arm but conferred limited cross-resistance to DTG. ${ }^{54,57,59,60}$

The Phase IIb VIKING trial examined the efficacy of DTG in HIV-infected patients harboring RAL-resistant strains, and compared $50 \mathrm{mg}$ once-daily or twice-daily dosing. By week $24,41 \%$ and $75 \%$ of participants in cohort I and II, respectively, had VLs $<50$ copies $/ \mathrm{mL}$ via the time to loss of virologic response (TLORV) algorithm. Integrase resistance was observed in $15 \%$ of all participants and was classified as RAL mutations. No single mutation emerged that appeared to significantly decrease DTG susceptibility based on the current knowledge of DTG resistance pathways. Basing on these findings, the $50 \mathrm{mg}$ twice-daily dose become the recommended dosing for individuals with prior RAL or EVG failure with integrase resistance mutations. ${ }^{55}$

In the single-arm, open-label, Phase III VIKING-3 study, antiretroviral-experienced, heavily treated participants with multidrug resistance, including RAL and EVG resistance, received DTG $50 \mathrm{mg}$ twice daily plus an optimized rescue drug combination. ${ }^{56}$

Overall, $69 \%$ of subjects achieved $\mathrm{VL}<50$ copies $/ \mathrm{mL}$ at week 24. Multivariate analyses demonstrated a strong association between baseline DTG susceptibility and response. Response was most reduced in subjects with Q148 plus two 
or more resistance-associated mutations. DTG $50 \mathrm{mg}$ twice daily was well tolerated, with low (3\%) discontinuation rate due to adverse events, similar to INSTI-naïve subjects receiving DTG $50 \mathrm{mg}$ once daily.

\section{Drug safety}

DTG has relatively few side effects and is generally well tolerated compared with most other available ARVs. ${ }^{59}$ Rate of adverse events leading to DTG discontinuation in most studies is $2 \%-3 \%$. The most common adverse reactions of moderate to severe intensity with an incidence of $\geq 2 \%$ in the clinical trials were insomnia and headache. Cases of hypersensitivity reactions were reported in $<1 \%$ of trial participants.

Renal elimination of unchanged DTG is less than $1 \%{ }^{6}$ In a comparison study of DTG versus RAL (SPRING), a small increase in creatinine was observed in the DTG arm. ${ }^{51}$ DTG decreases tubular secretion of creatinine without affecting glomerular function (measured by iohexol clearance), with increases in serum creatinine observed within the first 4 weeks of treatment (mean change in serum creatinine of $0.11 \mathrm{mg} / \mathrm{dL}$ after 48 weeks). ${ }^{51,62,63}$ This can be explained by the fact that DTG inhibits the renal organic transporter OCT2, which is involved in creatinine secretion. ${ }^{57,61}$

DTG displays a favorable metabolic profile, similar to RAL, as demonstrated in the SPRING-2 and SAILING trials. $^{51,54}$

\section{Drug resistance}

Integrase inhibitors represent a novel class of drugs completely active against viruses resistant to all other classes of drugs. ${ }^{47,64-66}$ Data on integrase inhibitor resistance come primarily from clinical trials and in vitro studies. Approximately one in six US patients undergoing INSTI genotyping resistance tests for clinical decision making harbors significant resistance, but DTG is likely to have full or partial activity against most variants observed ${ }^{65}$ RAL and EVG share a common clinical resistance profile, including primary resistance mutations, often in combination with secondary mutations that further compensate for the decreased fitness associated with the primary mutations. ${ }^{64}$

For RAL, major resistance mutations usually occur at positions Y143, N155, and Q148, whereas resistance to EVG is mostly associated with the emergence of mutations at positions T66, E92, N155, and Q148. ${ }^{66,67}$ The broad crossresistance profile between RAL and EVG precludes their sequential use in individuals failing either of them. ${ }^{47}$

In vitro studies showed that DTG has a resistance profile markedly distinct from that of RAL and EVG. The possibility of replacing RAL/EVG with DTG in heavily treatmentexperienced patients failing on RAL or EVG with viruses harboring resistance integrase mutations was evaluated in the VIKING trials. ${ }^{56}$ DTG demonstrated efficacy against most isolates resistant to RAL and EVG except for viruses containing mutations of the Q148HKR pathway, with at least one mutation among G140ACS, L74I, and E138AKT that conferred lower susceptibility to DTG.

Evidence from in vitro studies shows that DTG-resistant viruses are impaired in their ability to acquire further resistance to each of nevirapine and lamivudine as a consequence of their relative inability to develop resistance mutations associated with these two compounds. ${ }^{68}$

DTG is the only antiretroviral drug not yet associated with de novo emergence of resistance mutations in treatment-naïve individuals. ${ }^{47}$ However, data from in vitro studies and clinical trials suggest the possibility of cross-resistance between first- and second-generation INSTIs. ${ }^{64,66,67}$ In a clinical setting, few occurrences of virologic failure with resistance mutations have been documented in previously drug-experienced patients who received DTG as a first-time INSTI as a component of a second-line regimen. ${ }^{67}$ Even though these profiles are infrequent at the moment, they need to be monitored in all current patients treated with INSTIs; moreover, more datasets are needed to assess the long-term efficacy of DTG in patients failing on RAL and EVG. ${ }^{47,64}$

\section{Patient consideration}

Adherence is a well-known problem of great importance in HIV-infected patients on highly active antiretroviral therapy (HAART) and is influenced by several factors. Poor adherence leads to treatment failure by a selection of resistant viruses. ${ }^{69,70}$ Recent studies have underlined that durable viral suppression can be achieved also by regimens that require an adherence rate less than $95 \% .{ }^{71-73}$ Furthermore, the relationship between adherence and the development of resistance differs by drug class. ${ }^{74-76}$

Treatment simplification strategies improve patients' adherence to HAART. The reduced number of pills affects both the adherence and the quality of life (QoL) of HIV-infected patients on HAART, as many studies have shown. ${ }^{77-81}$ By substituting to a regimen of one pill once a day, an improvement can be observed for both adherence and QoL, while maintaining virological and immunological efficacy. On the other hand, the improvement of QoL influences the adherence. It can be said that HAART simplicity works in favor of the long-term success of therapy throughout adherence, QoL and subjective perception of health status, 
and these combined variables influence one another, enhancing the final result.

The fixed dose combination (FDC) regimen is the favorite from a patient's point of view in terms of tolerability, convenience, and simplicity and also in terms of efficacy. ${ }^{82}$ The complexity of the antiretroviral regimens is one of the most important barriers to HAART's adherence. The patients consider the number of daily pills, the frequency and timing of doses, the dietary restrictions, the adverse events, and the storage requirements to be the major factors that impair adherence to HAART. ${ }^{80,83}$

The presence of symptoms and the fear of changes to their appearance are associated with intentional nonadherence and significantly affect the perception of health status and self-reported QoL. The reduction in the proportion of subjects reporting any symptom has been observed after switching to FDR-based HAART, along with the increase in self-reported QoL. ${ }^{82}$ Patients indicate FDC-based HAART as the regimen of choice based on simplicity, convenience, and tolerability.

The results of the Italian ADONE study confirm that simpler regimens are associated with higher adherence rates as well as improvement in QoL. Adding the patients' preferences to these variables, the FDC regimen can assure long-lasting efficacy of HAART. ${ }^{82}$

Once-daily DTG with fixed-dose NRTIs in one pill represents the best available treatment for HIV-1-naïve patients and not only from the patient's point of view. The improved tolerability of the considered regimen among the existing others, the reduced drug interactions, the lack of food restrictions, and the higher genetic barrier in the class make DTG in FDC the most appealing treatment choice.

Looking at the patient consideration literature concerning INSTIs, we have found two clinical trials measuring patient satisfaction. ${ }^{84,85}$ In one of these studies, the effect of replacing EFV with RAL on patient preference, daytime sleepiness, sleep quality, anxiety, and lipid levels was investigated in a well-designed randomized, double-blind, crossover study. ${ }^{84}$ Patients who tolerated EFV, with less than 50 copies/mL HIV RNA, were randomized into two groups: the RAL-first group started with RAL (400 mg twice daily) and EFV placebo, and the EFV-first group with EFV (600 mg once daily) and RAL placebo. After 2 weeks, both groups switched to the alternative regimen. The primary end point was patient preference for the first or the second regimen, assessed after 4 weeks. Treatment preference of the 53 patients who completed the study was 22 for RAL and 12 for EFV, whereas 19 did not express a preference. A significant difference in anxiety and stress scores favoring $\operatorname{RAL}(P=0.04$ and 0.03 , respectively) was observed. In conclusion, half of patients previously on a stable EFV preferred to switch to RAL after double-blind exposure to RAL for 2 weeks. Substitution of EFV by RAL significantly impacted on lipid levels, stress, and anxiety scores.

A substudy of the FLAMINGO trial compared treatment satisfaction with DTG versus DRV/r-based regimens in ART-naïve subjects. ${ }^{85}$ Satisfaction with treatment was measured with the HIV Treatment Satisfaction Questionnaire (HIVTSQ), a ten-item self-reported scale that measures overall satisfaction with treatment and by specific domains such as convenience, ease of use, and flexibility. ${ }^{86}$ The HIVTSQ included 420 subjects from the US, France, Germany, Italy, and Spain, for which valid translations were available. Median HIVTSQ total scores were higher in the DTG treatment group compared with the DRV/r treatment group at all evaluated time points (4, 24, and 48 weeks); however, differences were statistically significant at week 24 $(P=0.005)$ only. The convenience item score was statistically significantly higher in the DTG group compared with the DRV/r treatment group at weeks $4(P<0.001), 24$ ( $P=0.002)$, and $48(P=0.003)$. These findings suggest that in addition to showing improvement in treatment efficacy, DTG showed improvements in treatment satisfaction over DRV/RTV.

\section{Conclusion}

DTG is a new-generation INSTI recently approved for the treatment of HIV-1-infected adult patients, with distinct advantages compared with other available antiretroviral agents, including the potential to improve patient adherence. DTG has a favorable pharmacokinetic profile with a prolonged intracellular half-life, which allows, in INSTInaïve patients, a once-daily dosing without the need for pharmacokinetic boosting and regardless of meals. Compared with RAL and EVG, DTG offers a favorable resistance profile and a higher genetic barrier to resistance. The safety profile of DTG is also encouraging, as the drug is expected to be associated with mild treatment-related side effects, minimal impact, if any, on lipids, and good tolerability, especially as compared with EFV-treated patients. ${ }^{87}$

In Phase III clinical trials, DTG-containing regimens have demonstrated either noninferiority or superiority to current firstline agents such as RAL-, DRV/r-, and EFV-containing regimens. This drug also exhibited efficacy in ART-experienced participants and may be an option when given twice daily in patients harboring resistance to RAL and EVG. 
Table 3 US guidelines - Department of Health and Human Services (May 2014).' Recommended initial combination regimens for all patients, regardless of initial viral load or CD4 count

\begin{tabular}{|c|c|c|}
\hline Regimens & Rating* & Notes \\
\hline \multicolumn{3}{|l|}{ NNRTI based } \\
\hline • EFV/TDF/FTC & $\mathrm{Al}$ & \\
\hline \multicolumn{3}{|l|}{ PI based } \\
\hline - ATV/r plus TDF/FTC & $\mathrm{Al}$ & \\
\hline - DRV/r plus TDF/FTC & $\mathrm{Al}$ & \\
\hline \multicolumn{3}{|l|}{ INSTI based } \\
\hline \multirow{2}{*}{ - DTG plus ABC/3TC } & $\mathrm{Al}$ & Only for patients \\
\hline & & HLA-B*570I negative \\
\hline • DTG plus TDF/FTC & $\mathrm{Al}$ & \\
\hline \multirow[t]{3}{*}{ - EVG/COBI/TDF/FTC } & $\mathrm{Al}$ & Only for patients \\
\hline & & with pretreatment \\
\hline & & $\mathrm{CrCl} \geq 70 \mathrm{~mL} / \mathrm{min}$ \\
\hline - RAL plus TDF/FTC & Al & \\
\hline
\end{tabular}

Note: *AI, strong recommendation from randomized clinical trials.

Abbreviations: NNRTI, nonnucleoside reverse transcriptase inhibitor; EFV, efavirenz; TDF, tenofovir; FTC, emtricitabine; PI, protease inhibitor; ATV/r, atazanavir + ritonavir; DRV/r, darunavir + ritonavir; INSTI, integrase strand transfer inhibitor; DTG, dolutegravir; ABC, abacavir; 3TC, lamivudine; EVG, elvitegravir; $\mathrm{COBI}$, cobicistat; RAL, raltegravir; $\mathrm{CrCl}$, creatinine clearance.

With its potent activity, good tolerability, simplicity of dosing, and minimal drug interaction profile, DTG will likely play a major role in the management of patients with HIV-1 infection.

On the basis of clinical trial data, current US and European guidelines endorse DTG in combination with NRTIs (either $\mathrm{ABC} / 3 \mathrm{TC}$ or TDF/FTC) as one of the recommended regimens in ART-naïve patients (Tables 3 and 4). ${ }^{1,2}$

Table 4 European AIDS Clinical Society (EACS) updated guidelines (November 20I4), modified. ${ }^{2}$ Recommended initial regimens for HIV-infected adult patients. A drug from column $A$ should be combined with the NRTI backbone listed in column B

\begin{tabular}{|c|c|c|}
\hline A & B & Notes \\
\hline NNRTI & NRTI & \\
\hline - EFV & $\mathrm{ABC} / 3 \mathrm{TC}$ or TDF/FTC* & $\begin{array}{l}\text { ABC/3TC, TDF/FTC, } \\
\text { EFV/TDF/FTC coformulated }\end{array}$ \\
\hline - $\mathrm{RPV} * *$ & $\mathrm{ABC} / 3 \mathrm{TC}$ or TDF/FTC & RPV/TDF/FTC coformulated \\
\hline \multicolumn{3}{|l|}{$\mathrm{Pl} / \mathrm{r}$} \\
\hline - ATV/r & $\mathrm{ABC} / 3 \mathrm{TC}$ or TDF/FTC & \\
\hline - $\mathrm{DRV} / \mathrm{r}$ & $\mathrm{ABC} / 3 \mathrm{TC}$ or TDF/FTC & \\
\hline \multicolumn{3}{|l|}{ INSTI } \\
\hline - $\mathrm{EVG}+\mathrm{COBI}$ & TDF/FTC & $\begin{array}{l}\text { TDF/FTC/EVG/COBI } \\
\text { coformulated }\end{array}$ \\
\hline - DTG & $\mathrm{ABC} / 3 \mathrm{TC}$ or TDF/FTC & $\begin{array}{l}\mathrm{ABC} / 3 \mathrm{TC} / \mathrm{DTG} \\
\text { coformulated }\end{array}$ \\
\hline - RAL & $\mathrm{ABC} / 3 \mathrm{TC}$ or TDF/FTC & \\
\hline
\end{tabular}

Notes: *ABC contraindicated if HLA-B*570I positive; **RPV: only if HIV viral load $<100,000$ copies/mL

Abbreviations: HIV, human immunodeficiency virus; NNRTI, nonnucleoside reverse transcriptase inhibitor; EFV, efavirenz; $A B C$, abacavir; $3 T C$, lamivudine; TDF, tenofovir; FTC, emtricitabine; EFV, efavirenz; RPV, rilpivirina; PI, protease inhibitor; ATV/r, atazanavir + ritonavir; DRV/r, darunavir + ritonavir; INSTI, integrase strand transfer inhibitor; COBI, cobicistat; DTG, dolutegravir; RAL, raltegravir.
Most of the favorable clinical experiences from clinical trials are based on a combination of DTG with $\mathrm{ABC} / 3 \mathrm{TC}$. DTG will be coformulated with $\mathrm{ABC} / 3 \mathrm{TC}$ as a single pill, once-daily regimen, and this will provide a further advantage, given that single tablet regimens are associated with higher adherence rates as well as improvement in QoL and enhanced patient preference. On the other hand, attention to the safety profile of the nucleoside backbone will be required, including HLAB $* 5701$ genetic testing, in order to identify patients at risk for $\mathrm{ABC}$-associated hypersensitivity reactions.

\section{Disclosure}

The authors report no conflicts of interest in this work.

\section{References}

1. Panel on Antiretroviral Guidelines for Adults and Adolescents. Guidelines for the Use of Antiretroviral Agents in HIV-1-Infected Adults and Adolescents. Department of Health and Human Services. Available at: http://aidsinfo.nih.gov/ContentFiles/AdultandAdolescentGL.pdf. Accessed January 27, 2015.

2. European AIDS Clinical Society. Guidelines. Clinical Management and Treatment of HIV Infected Adults in Europe. 7.1. November 2014. Available at: http://www.eacsociety.org/Portals/0/GUIDELINES/English\%20 PDF\%20-\%20Version\%207.1.pdf. Accessed January 27, 2015.

3. Cruciani M, Mengoli C, Malena M, et al. Virological efficacy of abacavir: systematic review and meta-analysis. J Antimicrob Chemother. 2014;69(12):3169-3180.

4. Rokx C, Fibriani A, van de Vijver DA, et al; for the AIDS Therapy Evaluation in the Netherlands (ATHENA) National Observational Cohort. Increased virological failure in naïve HIV-1-infected patients taking lamivudine compared with emtricitabine in combination with tenofovir and efavirenz or nevirapine in the Dutch Nationwide ATHENA Cohort. Clin Infect Dis. 2015;60(1):143-153.

5. Ford N, Hill A, Vitoria M, Mills EJ. Comparative efficacy of lamivudine and emtricitabine: comparing the results of randomized trials and cohorts. Clin Infect Dis. 2015;60(1):154-156.

6. Ford N, Shubber Z, Hill A, et al. Comparative efficacy of lamivudine and emtricitabine: a systematic review and meta-analysis of randomized trials. PLoS One. 2013;8:e79981.

7. Mallal S, Nolan D, Witt C, et al. Association between presence of HLA-B*5701, HLA-DR7, and HLA-DQ3 and hypersensitivity to HIV-1 reverse-transcriptase inhibitor abacavir. Lancet. 2002;359(9308): $727-732$.

8. Hetherington S, Hughes AR, Mosteller M, et al. Genetic variations in HLA-B region and hypersensitivity reactions to abacavir. Lancet. 2002;359(9312):1121-1122.

9. Mallal S, Phillips E, Carosi G, et al. HLA-B*5701 screening for hypersensitivity to abacavir. $N$ Engl J Med. 2008;358(6):568-579.

10. The DAD Study Group. Class of antiretroviral drugs and the risk of myocardial infarction. N Engl J Med. 2007;356(17):1723-1735.

11. Sabin CA, Worm SW, Weber R, et al. D:A:D Study Group. Use of nucleoside reverse transcriptase inhibitors and risk of myocardial infarction in HIV-infected patients enrolled in the D:A:D study: a multicohort collaboration. Lancet. 2008;371(9622):1417-1426.

12. Strategies for Management of Anti-Retroviral Therapy/INSIGHT; DAD Study Groups. Use of nucleoside reverse transcriptase inhibitors and risk of myocardial infarction in HIV-infected patients. AIDS. 2008;22(14):F17-F24

13. Worm SW, Sabin C, Weber R, et al. Risk of myocardial infarction in patients with HIV infection exposed to specific individual antiretroviral drugs from the 3 major drug classes: the data collection on adverse events of anti-HIV drugs (D:A:D) study. J Infect Dis. 2010;201(3):318-330. 
14. Aberg JA, Ribaudo H. Cardiac risk: not so simple. J Infect Dis. 2010; 201(3):315-317.

15. George E, Lucas GM, Nadkarni GN, Fine DM, Moore R, Atta MG. Kidney function and the risk of cardiovascular events in HIV-1-infected patients. AIDS. 2010;24(3):387-394.

16 Deeks JJ, Dinnes J, D’Amico R, et al. Evaluating non-randomised intervention studies. Health Technol Assess. 2003;7(27):iii-x, 1-173.

17. Brothers $\mathrm{CH}$, Hernandez JE, Cutrell AG, et al. Risk of myocardial infarction and abacavir therapy: no increased risk across 52 Glaxo Smith Kline sponsored clinical trials in adult subjects. J Acquir Immune Defic Syndr. 2009;51(1):20-28.

18. Cruciani M, Zanichelli V, Serpelloni G, et al. Abacavir use and cardiovascular disease events: a meta-analysis of published and unpublished data. AIDS. 2011;25(16):1993-2004.

19. Ding X, Andraca-Carrera E, Cooper C, et al. No association of abacavir use with myocardial infarction: findings of an FDA meta-analysis. J Acquir Immune Defic Syndr. 2012;61(4):441-447.

20. Lang S, Mary-Krause M, Cotte L, et al. Clinical Epidemiology Group of the French Hospital Database on HIV. Impact of individual antiretroviral drugs on the risk of myocardial infarction in human immunodeficiency virus-infected patients: a case-control study nested within the French Hospital Database on HIV ANRS cohort CO4. Arch Intern Med. 2010;170(14):1228-1238.

21. Bedimo RJ, Westfall AO, Drechsler H, Vidiella G, Tebas P. Abacavir use and risk of acute myocardial infarction and cerebrovascular events in the highly active antiretroviral therapy era. Clin Infect Dis. 2011;53(1):84-91.

22. Ribaudo HJ, Benson CA, Zheng Y, et al; for the ACTG A5001/ALLRT Protocol Team. No risk of myocardial infarction associated with initial antiretroviral treatment containing abacavir: short and long-term results from ACTG A5001/ALLRT. Clin Infect Dis. 2011;52(7):929-940.

23. Aberg JA. Cardiovascular complications in HIV management: past, present, and future. J Acquir Immune Defic Syndr. 2009;50(1):54-64.

24. Moyle GJ, Stellbrink HJ, Compston J, et al. 96-week results of abacavir/lamivudine versus tenofovir/emtricitabine, plus efavirenz, in antiretroviral-naïve, HIV-1-infected adults: ASSERT study. Antivir Ther. 2013;18(7):905-913.

25. Sax PE, Tierney C, Collier AC, et al. Abacavir-lamivudine versus tenofovir-emtricitabine for initial HIV-1 therapy. $N$ Engl J Med. 2009;361(23):2230-2240.

26. Smith KY, Patel P, Fine D, et al. Randomized, double-blind, placebomatched, multicenter trial of abacavir/lamivudine or tenofovir/ emtricitabine with lopinavir/ritonavir for initial HIV treatment. AIDS. 2009;23(12):1547-1556.

27. Pappa K, Hernandez J, Ha B, et al. Abacavir/lamivudine shows robust virologic responses in ART-naïve patients for baseline (BL) viral loads of $>100,000 \mathrm{c} / \mathrm{mL}$ and $<100,000 \mathrm{c} / \mathrm{mL}$ by endpoint used in ACTG5202. In: Abstracts of the Seventeenth International AIDS Conference, Mexico City, 2008. Abstract THAB0304.

28. Tan DH, Chan K, Raboud J, et al. Comparison of abacavir/lamivudine and tenofovir/emtricitabine among treatment-naïve HIV-infected patients initiating therapy. J Acquir Immune Defic Syndr. 2011;58(1):38-46.

29. Walmsley SL, Antela A, Clumeck N, et al. Dolutegravir plus abacavir-lamivudine for the treatment of HIV-1 infection. N Engl J Med. 2013;369(19):1807-1818

30. Powderly WG. Integrase inhibitors in the treatment of HIV-1 infection. $J$ Antimicrob Chemother. 2010;65(12):2485.

31. Delelis O, Carayon K, Saib A, Deprez E, Mouscadet JF. Integrase and integration: biochemical activities of HIV-1 integrase. Retrovirology. 2008;5:114.

32. Hazuda DJ, Felock P, Witmer M, et al. Inhibitors of strand transfer that prevent integration and inhibit HIV-1 replication in cells. Science. 2000;287(5453):646-650.

33. Mesplède T, Quashie PK, Zanichelli V, Wainberg MA. Integrase strand transfer inhibitors in the management of HIV-positive individuals. Ann Med. 2014;46(3):123-129.
34. Hicks C, Gulick RM. Raltegravir: the first HIV type 1 integrase inhibitor. Clin Infect Dis. 2009;48(7):931-939.

35. Steigbigel RT, Cooper DA, Teppler H, et al. Long-term efficacy and safety of raltegravir combined with optimized background therapy in treatmentexperienced patients with drug-resistant HIV infection: week 96 results of the BENCHMRK 1 and 2 phase III trials. Clin Infect Dis. 2010;50(4):605-612.

36. Steigbigel RT, Cooper DA, Kumar PN, et al. Raltegravir with optimized background therapy for resistant HIV-1 infection. N Engl J Med. 2008;359(4):339-354.

37. Lennox JL, DeJesus E, Lazzarin A, et al. Safety and efficacy of raltegravir-based versus efavirenz-based combination therapy in treatment-naïve patients with HIV-1 infection: a multicentre, doubleblind randomised controlled trial. Lancet. 2009;374(9692):796.

38. Rockstroh JK, Lennox JL, DeJesus E, et al. Long-term treatment with raltegravir or efavirenz combined with tenofovir/emtricitabine for treatment-naïve human immunodeficiency virus-1-infected patients: 156-week results from STARTMRK. Clin Infect Dis. 2011;53(8): 807-816.

39. Eron JJ, Cooper DA, Steigbigel RT, et al. Efficacy and safety of raltegravir for treatment of HIV for 5 years in the BENCHMRK studies: final results of two randomised, placebo-controlled trials. Lancet Infect Dis. 2013;13(7):587-596.

40. Markowitz M, Nguyen BY, Gotuzzo E, et al. Sustained antiretroviral effect of raltegravir after 96 weeks of combination therapy in treatmentnaïve patients with HIV-1 infection. J Acquir Immune Defic Syndr. 2009;52(3):350-356.

41. Markowitz M, Nguyen BY, Gotuzzo E, et al. Rapid and durable antiretroviral effect of the HIV-1 integrase inhibitor raltegravir as part of combination therapy in treatment-naïve patients with HIV-1 infection: results of a 48-week controlled study. J Acquir Immune Defic Syndr. 2007;46(2):125-133.

42. Sax PE, DeJesus E, Mills A, et al. Co-formulated elvitegravir, cobicistat, emtricitabine, and tenofovir versus coformulated efavirenz, emtricitabine, and tenofovir for initial treatment of HIV-1 infection: a randomised, double-blind, phase 3 trial, analysis of results after 48 weeks. Lancet. 2012;379(9835):2439-2448.

43. Zolopa A, Sax PE, DeJesus E, et al. A randomized double-blind comparison of coformulated elvitegravir/cobicistat/emtricitabine/tenofovir disoproxil fumarate versus efavirenz/emtricitabine/tenofovir disoproxil fumarate for initial treatment of HIV-1 infection: analysis of week 96 results. J Acquir Immune Defic Syndr. 2013;63(1):96-100.

44. DeJesus E, Rockstroh JK, Henry K, et al. Co-formulated elvitegravir, cobicistat, emtricitabine, and tenofovir disoproxil fumarate versus ritonavir-boosted atazanavir plus co-formulated emtricitabine and tenofovir disoproxil fumarate for initial treatment of HIV-1 infection: a randomised, double-blind, phase 3, non-inferiority trial. Lancet. 2012;379(9835):2429-2438.

45. Shah BM, Schafer J, Priano J, Squires KE. Cobicistat: a new boost for the treatment of human immunodeficiency virus infection. Pharmacotherapy. 2013;33(10):1107-1116.

46. Gilead Sciences, Inc. Stribild (elvitegravir/cobicistat/tenofovir/ emtricitabine) [package insert]. Foster City, CA; 2012.

47. Mesplede T, Quashie PK, Wainberg MA. Resistance to HIV integrase inhibitors. Curr Opin HIV AIDS. 2012;7:401-408.

48. van Lunzen J, Maggiolo F, Arribas J, et al. Once daily dolutegravir (S/GSK1349572) in combination therapy in antiretroviral-naïve adults with HIV: planned interim 48 week results from SPRING-1, a doseranging, randomised, phase $2 \mathrm{~b}$ trial. Lancet Infect Dis. 2012;12(2): 111-118.

49. Stellbrink H, Reynes J, Lazzarin A, et al. Dolutegravir in antiretroviralnaïve adults with HIV-1: 96-week results from a randomized doseranging study. AIDS. 2013;27:1771-1778.

50. Walmsley S, Antela A, Clumeck N, et al. Dolutegravir plus abacavirlamivudine for the treatment of HIV-1 infection. $N$ Engl $J$ Med. 2013;369(19):1807-1818. 
51. Raffi F, Rachlis A, Stellbrink HJ, et al. Once-daily dolutegravir versus raltegravir in antiretroviral-naïve adults with HIV-1 infection: 48 week results from the randomised, double-blind, non-inferiority SPRING-2 study. Lancet. 2013;381(9868):735-743.

52. Raffi F, Jaeger H, Quiros-Roldan E, et al. Once-daily dolutegravir versus twice-daily raltegravir in antiretroviral-naïve adults with HIV-1 infection (SPRING-2 study): 96 week results from a randomised, doubleblind, non-inferiority trial. Lancet Infect Dis. 2013;13(11):927-935.

53. Clotet B, Feinberg J, van Lunzen J, et al. Once-daily dolutegravir versus darunavir plus ritonavir in antiretroviral-naïve adults with HIV-1 infection (FLAMINGO): 48 week results from the randomised open-label phase 3b study. Lancet. 2014;383(9936):2222-2231.

54. Cahn P, Pozniak AL, Mingrone H, et al. Dolutegravir vs raltegravir in antiretroviral-experienced, integrase-inhibitor-naïve adults with HIV: week 48 results from the randomised, double-blind, non-inferiority SAILING study. Lancet. 2013;382(9893):700-708.

55. Eron JJ, Clotet B, Durant J, et al. Safety and efficacy of dolutegravir in treatment-experienced subjects with raltegravir resistant HIV type 1 infection: 24-week results of the VIKING study. J Infect Dis. 2013; 207(5):740-748.

56. Castagna A, Maggiolo F, Penco G, et al. Dolutegravir in antiretroviralexperienced patients with raltegravir- and/or elvitegravir-resistant HIV-1: 24-week results of the phase III VIKING-3 study. J Infect Dis. 2014;210(3):354-362.

57. Fernandez-Montero JV, Barreiro P, Labarga P, De Mendoza C, Soriano V. Dolutegravir, abacavir and lamivudine as HIV therapy. Expert Opin Pharmacother. 2014;15(7):1051-1057.

58. Raffi F, Pozniak A, Wainberg M. Has the time come to abandon efavirenz for first-line antiretroviral therapy? J Antimicrob Chemother. 2014;69(7):1742-1747.

59. Osterholzer DA, Goldman M. Dolutegravir: a next-generation integrase inhibitor for treatment of HIV infection. Clin Infect Dis. 2014;59(2): 265-271.

60. Temesgen Z, Talwani R, Rizza SA. Dolutegravir, an HIV integrase inhibitor for the treatment of HIV infection. Drugs Today (Barc). 2014; 50(1):7-14.

61. Reese MJ, Savina PM, Generaux GT, et al. In vitro investigations into the roles of drug transporters and metabolizing enzymes in the disposition and drug interactions of dolutegravir, a HIV integrase inhibitor Drug Metab Dispos. 2013;41(2):353-361.

62. Koteff J, Borland J, Chen S, et al. A phase 1 study to evaluate the effect of dolutegravir on renal function via measurement of iohexol and paraaminohippurate clearance in healthy subjects. Br J Clin Pharmacol. 2013;75(4):990-996.

63. Maggi P, Montinaro V, Rusconi S, et al. The problem of renal function monitoring in patients treated with the novel antiretroviral drugs. HIV Clin Trials. 2014;15:87-91.

64. Malet I, Gimferrer Arriaga L, Artese A, et al. New raltegravir resistance pathways induce broad cross-resistance to all currently used integrase inhibitors. J Antimicrob Chemother. 2014;69(8):2118-2122.

65. Hurt CB, Sebastian J, Hicks CB, Eron JJ. Resistance to HIV integrase strand transfer inhibitors among clinical specimens in the United States, 2009-2012. Clin Infect Dis. 2014;58(3):423-431.

66. Mesplède T, Quashie PK, Wainberg MA. Resistance to HIV integrase inhibitors. Curr Opin HIV AIDS. 2012;7(5):401-408.

67. Blanco JL, Varghese V, Rhee SY, Gatell JM, Shafer RW. HIV-1 integrase inhibitor resistance and its clinical implications. J Infect Dis. 2011;203(9):1204-1214

68. Oliveira M1, Mesplède T, Quashie PK, Moïsi D, Wainberg MA Resistance mutations against dolutegravir in HIV integrase impair the emergence of resistance against reverse transcriptase inhibitors. AIDS 2014;28(6):813-819.

69. Rodriguez-Rosado R, Jimenez-Nacher I, Soriano V, et al. Virological failure and adherence to antiretroviral therapy in HIV-infected patients. AIDS. 1998;12(9):1112-1113.
70. Bangsberg DR, Charlebois ED, Grant RM, et al. High levels of adherence do not prevent accumulation of HIV drug resistance mutations. AIDS. 2003;17(13):1925-1932.

71. Maggiolo F, Ravasio L, Ripamonti D, et al. Similar adherence rates favour different virologic outcomes for patients treated with nonnucleoside analogues or protease inhibitors. Clin Infect Dis. 2005;40(1) $158-163$.

72. Bangsberg D. Less than $95 \%$ adherence to nonnucleoside reverse transcriptase inhibitor therapy can lead to viral suppression. Clin Infect Dis. 2006;43(7):939-941.

73. Shah B, Walshe L, Saple DG, et al. Adherence to antiretroviral therapy and virologic suppression among HIV-infected persons receiving care in private clinics in Mumbai, India. Clin Infect Dis. 2007;44(9): 1235-1244.

74. Maggiolo F, Airoldi M, Kleinloog HD, et al. Effect of adherence to HAART on virologic outcome and on the selection of resistanceconferring mutations in NNRTI- or PI-treated patients. HIV Clin Trials. 2007;8(5):282-292.

75. Bangsberg DR, Acosta EP, Gupta R, et al. Adherence-resistance relationship for protease and non-nucleoside reverse transcriptase inhibitors explained by virological fitness. AIDS. 2006;20(2):223-231.

76. Bangsberg DR, Porco TC, Kagay C, et al. Modelling the HIV protease inhibitor adherence-resistance curve by use of empirically derived estimates. J Infect Dis. 2004;190(1):162-165.

77. Maggiolo F, Ripamonti D, Arici C, et al. Simpler regimens may enhance adherence to antiretrovirals in HIV infected patients. HIV Clin Trials. 2002;3(5):371-378.

78. Trotta MP, Ammassari A, Cozzi-Lepri A, et al. Adherence to highly active antiretroviral therapy is better in patients receiving non-nucleoside reverse transcriptase inhibitor-containing regimens than those receiving protease inhibitor-containing regimens. AIDS. 2003;17(7): 1099-1102.

79. Flandre P, Peytavin G, Meifreddy V, et al. Adherence to antiretroviral therapy and outcomes in HIV infected patients enrolled in an induction/ maintenance randomized trial. Antivir Ther. 2002;7(2):113-121.

80. Stone VE, Jordan J, Tolson J, et al. Perspectives on adherence and simplicity of HIV-infected patients on antiretroviral therapy. Selfreport of the relative importance of multiple attributes of Highly Active Antiretroviral Therapy (HAART) regimens in predicting adherence. J Acquir Immune Defic Syndr. 2004;36(3):808-816.

81. Boyle B, Jayaweera D, Witt MD, et al. Randomization to once-daily stavudine extended release/lamivudine/efavirenz versus more frequent regimen improves adherence while maintaining viral suppression. HIV Clin Trials. 2008;9(3):164-176.

82. Airoldi M, Zaccarelli M, Bisi L, et al. One-pill once-a-day HAART: a simplification strategy that improves adherence and quality of life of HIV-infected subjects. Patient Prefer Adherence. 2010;4:115-125.

83. Glass TR, De Geest S, Weber R, et al. Correlates of self-reported nonadherence to antiretroviral therapy in HIV-infected patients. The Swiss HIV Cohort study. J Acquir Immune Defic Syndr. 2006;41(3):385-392.

84. Nguyen A, Calmy A, Delhumeau C, et al. A randomized cross-over study to compare raltegravir and efavirenz (SWITCH-ER study). AIDS. 2011;25(12):1481-1487.

85. Murray M, Goodqin B, Hagins D, Gallien S, Brennan C, Min S. Measuring patient satisfaction with HIV treatments: comparing dolutegravir with darunavir/ $\mathrm{r}$ in the FLAMINGO study. Presented at: 20th International AIDS Conference; July 20-25, 2014; Melbourne, Australia. Poster WEPE065.

86. Woodcock A, Bradley C. Validation of the revised 10-item HIV Treatment Satisfaction Questionnaire status version and new change version. Value Health. 2006;9(5):20-33.

87. Wu G, Abraham T, Saad N. Dolutegravir for the treatment of adult patients with HIV-1 infection. Expert Rev Anti Infect Ther. 2014;12(5): $535-544$. 
Patient Preference and Adherence

Dovepress

\section{Publish your work in this journal}

Patient Preference and Adherence is an international, peer-reviewed, open access journal that focuses on the growing importance of patient preference and adherence throughout the therapeutic continuum. Patient satisfaction, acceptability, quality of life, compliance, persistence and their role in developing new therapeutic modalities and compounds to optimize

clinical outcomes for existing disease states are major areas of interest for the journal. This journal has been accepted for indexing on PubMed Central. The manuscript management system is completely online and includes a very quick and fair peer-review system, which is all easy to use. Visit http://www. dovepress.com/testimonials.php to read real quotes from published authors.

Submit your manuscript here: http://www.dovepress.com/patient-preference-and-adherence-journal 